\title{
Prostate cancer among different racial groups in the Western Cape: Presenting features and management
}

\author{
C F Heyns, M Fisher, A Lecuona, A van der Merwe
}

Objectives. We aimed to compare the presenting features and management of prostate cancer among different racial groups.

Patients and methods. We studied all patients diagnosed with prostate cancer at the Urological Oncology Clinic, Tygerberg Hospital, from January 1995 to December 2005. Most presented symptomatically as PSA screening is not readily available in the referral area of the hospital. Race was self-defined as white, coloured or black. Statistical analysis was performed using Student's $t$-test or Fisher's exact test, where appropriate. A two-tailed $p$-value $<0.05$ was accepted as statistically significant.

Results. There were 901 patients: 291 (32.3\%) white, 539 (59.8\%) coloured and $71(7.9 \%)$ black. Mean age at presentation was significantly higher in the white than the coloured and black groups (69.7, 67.9 and 68.9 years, respectively). Grade 1 adenocarcinoma was most common in the white (37\%) and coloured groups (38\%), and grade 2 was most common in the black group (39\%). There was a significantly lower percentage of patients with T3-4 disease at diagnosis in the white group (47\%) than the coloured (61\%) and black (62\%) groups. Mean serum PSA at diagnosis was significantly higher in the black than the coloured and white groups (766.1, 673.3 and $196.1 \mathrm{ng} / \mathrm{ml}$, respectively). Potentially curative therapy (radical prostatectomy or radiotherapy) was chosen by $31 \%$ of white, $23 \%$ of coloured and only $12 \%$ of black patients. The mean duration of follow-up was significantly shorter in the black than in the white or coloured groups (24.0, 31.5 and 35.0 months, respectively).

Conclusions. Black men presented with higher grade and stage disease and higher serum PSA, received potentially curative treatment less often, and had a shorter follow-up (probably owing to shorter survival) than the white and coloured groups. Greater prostate cancer awareness and education among patients and physicians and more widespread use of PSA screening of presymptomatic men at risk of prostate cancer is needed.

S Afr Med J 2011;101:267-270.
The incidence of prostate cancer varies worldwide, with the highest rates reported in the USA, and the lowest in parts of Asia. Prostate cancer incidence rates are 1.6 - 1.9 times higher, and mortality rates 2 - 3 times higher, among African-American men (AAM) than Caucasian-American men (CAM). ${ }^{1-6}$ AAM are younger at diagnosis, have higher grade and stage tumours and a worse prognosis, which may be due to greater biological aggressiveness. ${ }^{5,7,8}$

South Africa's population diversity offers an opportunity to compare disease patterns among different racial or ethnic groups living in the same geographical area. The population of Western Cape Province comprises white immigrants from Europe, blacks originally from central Africa, and coloureds, which refers to the group descended predominantly from indigenous Khoi and San inhabitants, with genetic input from subsequent European and Asian immigrants. ${ }^{9,10}$

Earlier studies suggested that prostate cancer was rare among indigenous blacks compared with whites living in Africa. ${ }^{11}$ However, the low incidence rates reported in African countries may be due to under-diagnosis and under-reporting, as a result of poorer access to screening and diagnostic facilities, or lack of national cancer registries. Some African countries have high incidence rates of prostate cancer, probably attributable to improved prostate-specific antigen (PSA) testing and diagnostic facilities and increased life expectancy. ${ }^{7,9}$

Department of Urology, Stellenbosch University and Tygerberg Hospital, Western Cape

C F Heyns, MMed (Urol), PhD, FCSSA (Urol)

M Fisher, FC Urol SA, MMed (Urol)

A Lecuona, FC Urol SA, MMed (Urol)

A van der Merwe, FC Urol SA, MMed (Urol)
Because of the extensive literature on racial differences in prostate cancer among men in the USA and the lack of such studies in African countries, we aimed to compare the presenting features and management of prostate cancer in men of different race in the Western Cape.

\section{Patients and methods}

We analysed the data on all men with a diagnosis of prostate cancer seen at the Urological Oncology Clinic of Tygerberg Hospital (a tertiary level public sector hospital) from January 1995 to December 2005. Most patients were referred from primary health care facilities in Western Cape Province (where PSA screening of asymptomatic men is not readily available) and almost all presented because of prostate cancer-related symptoms.

The patient's race was self-assigned as white, coloured or black. The cancers were clinically staged according to the 2002 TNM classification. Clinical tumour stage was determined by digital rectal examination $(\mathrm{DRE})$ as $\mathrm{T} 1=$ (clinically benign), $\mathrm{T} 2=$ palpable cancer confined to the prostate, $\mathrm{T} 3$ = invasion of the prostatic capsule, or T4 = involvement of peri-prostatic tissues. Haematogenous metastases were detected by radiography or skeletal scintigraphy.

The diagnosis of prostate cancer was confirmed by histological examination of specimens obtained by transrectal biopsy or transurethral resection of the prostate (TURP). Indications for prostate biopsy included a serum PSA $>4 \mathrm{ng} / \mathrm{ml}$ or a suspicious DRE. A clinical diagnosis (without histological confirmation) was made in some patients, based on clinical stage T3/4 prostate cancer on DRE, serum PSA $>50 \mathrm{ng} / \mathrm{ml}$, or sclerotic bone metastases on pelvic and vertebral X-rays. ${ }^{12}$ Histological grading was performed according to the MD Anderson system, which distinguishes between grades 1, 2 and 3: well, moderately and poorly differentiated.

Treatment decisions were taken at a weekly urology/oncology meeting where treatment options were discussed with the patient and his family. The final decision about treatment was taken by the patient in consultation with his family. 
Relevant details in the clinical records were prospectively entered by a single data capturer on an Excel database. These were analysed in 2009, allowing a minimum potential follow-up of 3 years for patients seen up to December 2005. Statistical analysis was performed using GraphPad Instat software. Comparison of means was performed using Student's $t$-test for parametric data and the Mann-Whitney test for non-parametric data. Fisher's exact test was used for contingency table analysis. A two-tailed $p$-value $<0.05$ was accepted as statistically significant.

\section{Results}

The study included 901 patients: 291 (32.3\%) white, 539 (59.8\%) coloured and 71 (7.9\%) black, which reflects the demography of patients seen at Tygerberg Hospital.

Mean age at presentation was significantly higher in the white than the coloured and black groups (69.7, 67.9 and 68.9 years, respectively). The peak age incidence was $80-89$ years in the white group, and $70-79$ years in the coloured and black groups (Fig. 1).

Among patients where the histological grading was available, grade 1 was the most common grade in the white (37\%) and coloured groups (38\%), and grade 2 the most common in the black group (39\%) (Fig. 2).

The most common clinical tumour stage at presentation was T1-2 in white patients $(53 \%)$ and T3-4 in the coloured (61\%) and black groups (62\%) (Fig. 3).

In patients with known M-stage, metastatic disease was most common in the black (53\%) and least common in the white group (28\%) (Fig. 4).

Mean and median serum PSA values at presentation were significantly higher in the black and coloured than the white group.

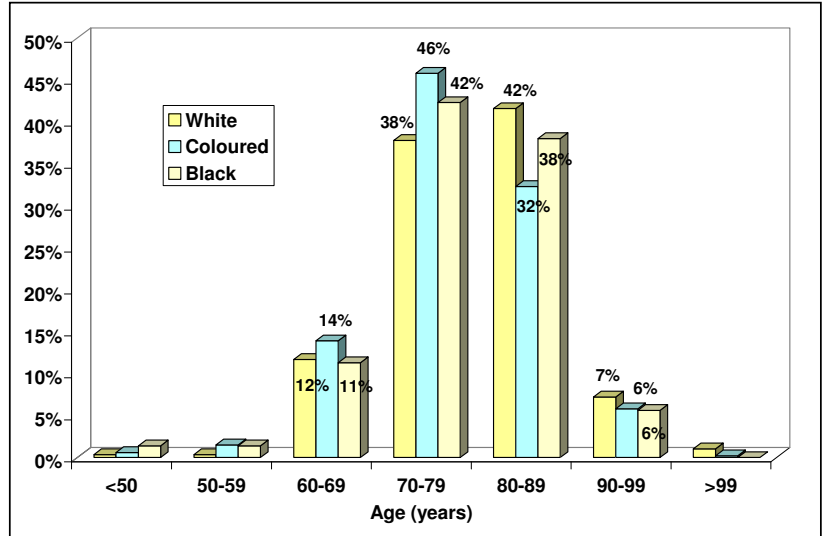

Fig. 1. Age distribution according to race.

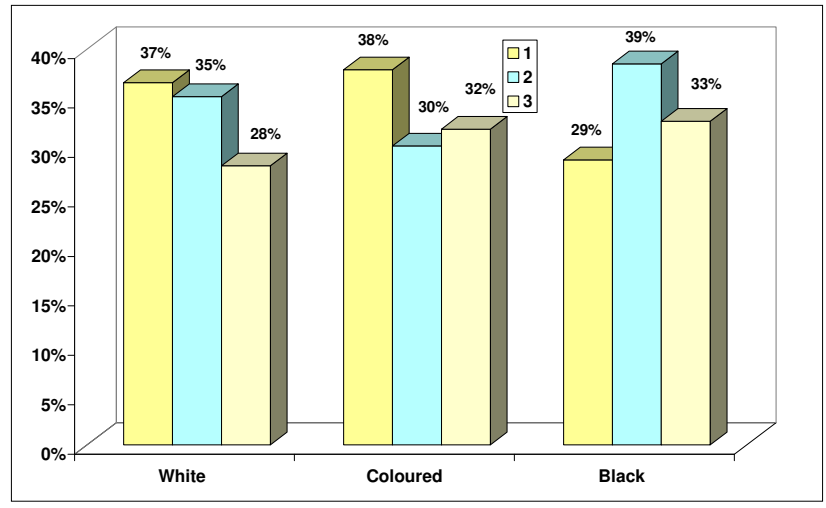

Fig. 2. Histological grade at presentation $(1=$ well, $2=$ moderately, $3=$ poorly differentiated).

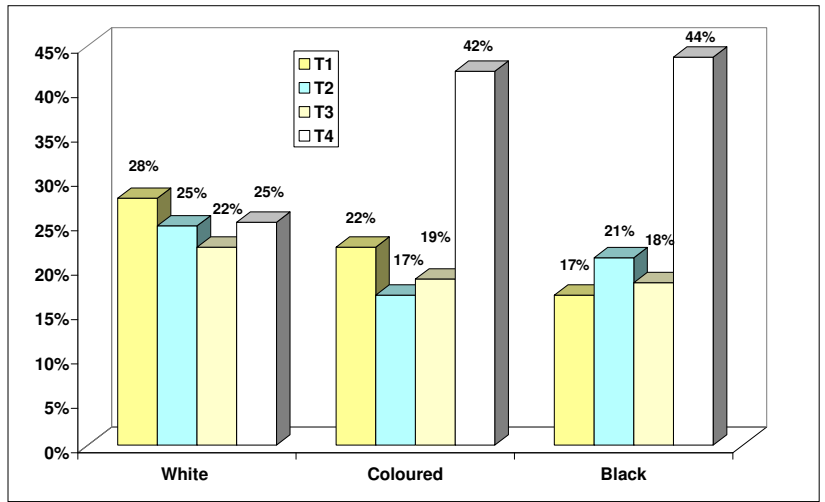

Fig. 3. Tumour stage at presentation.

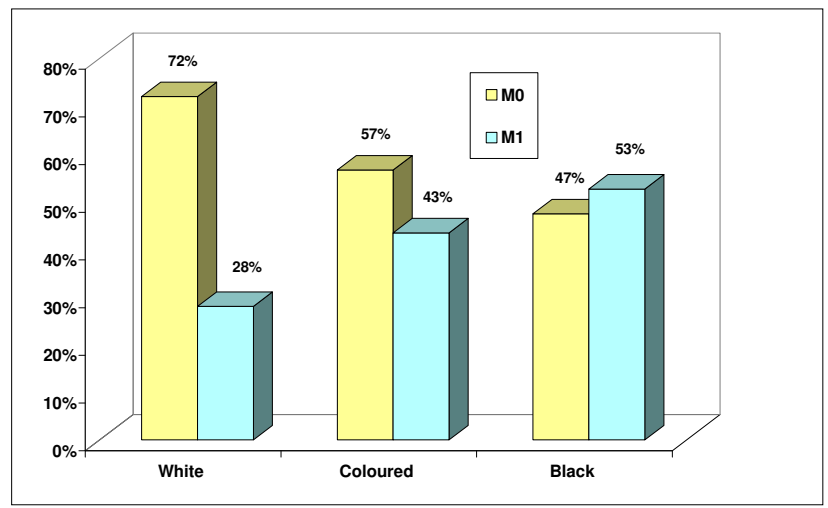

Fig. 4. Metastases (M-stage) at presentation.

There was a marked difference between the mean and median PSA values in all three groups, owing to a few extremely high outlier PSA values (Table I). Mean serum PSA was significantly increased in higher grade and higher stage cancer (Table I).

Over half of the patients, irrespective of race, received androgen deprivation therapy (ADT) as first-line treatment (Fig. 5). Watchful waiting (WW) was the second most common management choice in black patients. Potentially curative therapy was given in $31 \%$ of white, $23 \%$ of coloured and only $12 \%$ of black patients (Fig. 5).

Among men aged $<70$ years with clinically localised cancer (T1-2 M0) in whom potentially curative therapy is indicated, radical

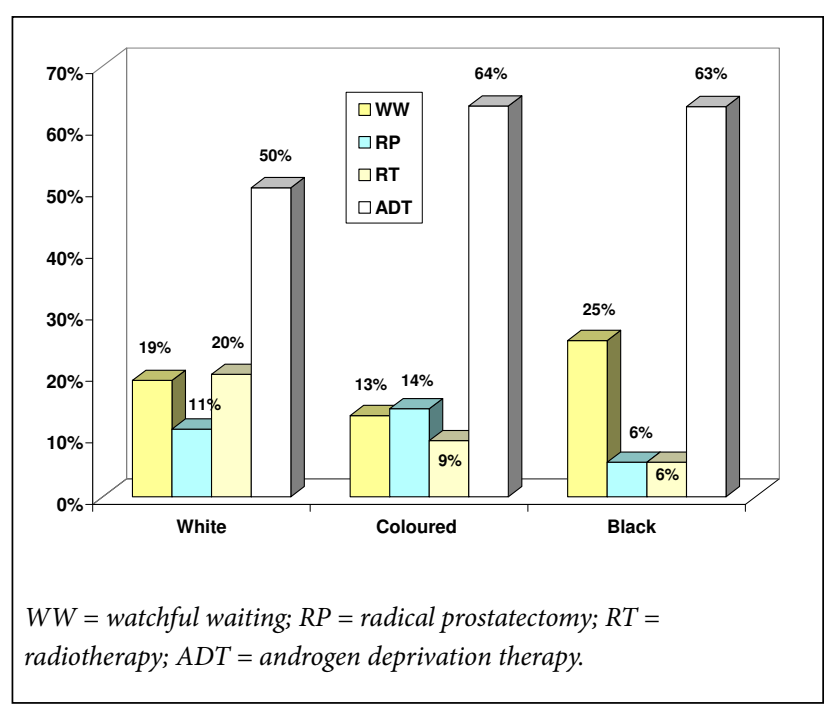

Fig. 5. Initial treatment. 
Table I. Serum PSA (ng/ml) at presentation according to race, histological grade and clinical stage

\begin{tabular}{|c|c|c|c|c|}
\hline & \multicolumn{4}{|c|}{ Race } \\
\hline & All & White & Coloured & Black \\
\hline Mean & 526.3 & $196.1^{*}$ & $673.3^{* \dagger}$ & $766.1^{\star \dagger}$ \\
\hline Median & 33.5 & 19.6 & 42.5 & 105 \\
\hline Minimum & 0.6 & 1 & 0.6 & 0.7 \\
\hline \multirow[t]{3}{*}{ Maximum } & 36213 & 6000 & 36213 & 19120 \\
\hline & \multicolumn{4}{|c|}{ Grade } \\
\hline & 1 & 2 & 3 & Unknown \\
\hline Mean & $206.8^{* \dagger}$ & $407.3^{* \dagger}$ & $802.2^{*}$ & $769.7^{\dagger}$ \\
\hline Median & 13.0 & 31.3 & 124.5 & 50.2 \\
\hline Minimum & 0.6 & 1.0 & 1.0 & 0.7 \\
\hline \multirow[t]{3}{*}{ Maximum } & 19120 & 9600 & 22600 & 36213 \\
\hline & \multicolumn{4}{|c|}{ Stage } \\
\hline & $\mathrm{T} 1$ & $\mathrm{~T} 2$ & $\mathrm{~T} 3$ & $\mathrm{~T} 4$ \\
\hline Mean & $16.0^{*}$ & $59.0^{*}$ & $226.1^{*}$ & $1269.2^{*}$ \\
\hline Median & 9.0 & 12.2 & 52.0 & 242.0 \\
\hline Minimum & 0.6 & 1.0 & 1.0 & 2.0 \\
\hline \multirow[t]{3}{*}{ Maximum } & 218 & 4349 & 6000 & 36213 \\
\hline & \multicolumn{4}{|c|}{ Stage } \\
\hline & N0 & $\mathrm{N}+$ & M0 & $\mathrm{M}+$ \\
\hline Mean & $99.9^{*}$ & $1750.5^{\star}$ & $93.7^{\dagger}$ & $1689.8^{\dagger}$ \\
\hline Median & 12.3 & 276.0 & 19.0 & 319.0 \\
\hline Minimum & 6.0 & 6.1 & 1.0 & 3.0 \\
\hline Maximum & 2802 & 12080 & 6600 & 36213 \\
\hline $\begin{array}{l}* \text { and } \dagger \text { denote } \\
\text { category marke }\end{array}$ & $\begin{array}{l}\text { S which ar } \\
\text { h the sam }\end{array}$ & $\begin{array}{l}\text { icantly differe } \\
l(p<0.05)\end{array}$ & om other values & the same \\
\hline
\end{tabular}

prostatectomy (RP) was chosen by $31.9 \%, 42.5 \%$ and $15 \%$ of white, coloured and black patients, respectively, whereas radiotherapy (RT) was chosen by $31.9 \%, 20.6 \%$ and $15 \%$ of the same groups, respectively. The remainder opted for WW or ADT.

In men aged $>70$ years with locally advanced or metastatic cancer (T3-4 or M1) in whom only palliative or deferred treatment is indicated, ADT was used in $66.7 \%, 85 \%$ and $86.3 \%$, and WW was chosen by $17.4 \%, 8.2 \%$ and $9.8 \%$ of white, coloured and black patients, respectively.

Patients were most often lost to follow-up with no reason available, and were known to have died in $11 \%$ of the white and coloured groups, and in only $1.4 \%$ of the black group. The mean duration of follow-up was significantly shorter in the black than in the white or coloured groups, and it was also shorter in high-grade than low-grade cancer, and in higher clinical stage compared with lower stage cancer (Table II).

\section{Discussion}

In the USA, the average age of AAM at presentation with prostate cancer is approximately 3 years younger than that of CAM. ${ }^{3}$ The peak age at diagnosis of prostate cancer in African countries is on average a decade earlier than in developed countries. ${ }^{9}$ However, Senegalese men diagnosed with prostate cancer had a significantly higher mean age than AAM in the USA ( $69 \mathrm{v} .61$ years). The probable explanation is that most men in the USA were diagnosed earlier by
Table II. Duration of follow-up (months) according to race, histological grade and clinical stage

\begin{tabular}{lllll}
\hline & All & White & Coloured & Black \\
\hline Mean & 32.1 & $35.0^{*}$ & $31.5^{\dagger}$ & $24.0^{* \dagger}$ \\
Median & 21.0 & 24.0 & 21.0 & 14.0 \\
Minimum & 1.0 & 1.0 & 1.0 & 1.0 \\
Maximum & 173 & 169 & 173 & 101 \\
& G1 & G3 & T1 & T2 \\
\hline Mean & $43.4^{*}$ & $36.5^{*}$ & $34.9^{\dagger}$ & 39.0 \\
Median & 35.0 & 27.0 & 26.5 & 27.0 \\
Minimum & 1.0 & 1.0 & 1.0 & 1.0 \\
Maximum & 169 & 173 & 141 & 152 \\
& T3 & T4 & M0 & M1 \\
\hline Mean & 35.4 & $24.8^{\dagger}$ & $47.4^{\ddagger}$ & $24.4^{\ddagger}$ \\
Median & 25.0 & 18.0 & 42.5 & 18.0 \\
Minimum & 1.0 & 1.0 & 1.0 & 1.0 \\
Maximum & 169 & 173 & 173 & 137 \\
* † and $¥$ denote values which are significantly different from other values in the same \\
category marked with the same symbol $(p<0.05)$. & & \\
\hline
\end{tabular}

PSA screening, whereas Senegalese men were most often diagnosed later with prostate cancer-related symptoms. ${ }^{4}$

In the present study, the mean age of the coloured and black groups was slightly but significantly lower than that of the white group, and the peak age at presentation was a decade earlier (Fig. 1). The peak age at presentation depends on life expectancy of the population, which is considerably lower in most African countries than in the USA. The incidence of prostate cancer increases progressively with age; therefore, in a population with a longer life expectancy and greater numbers of older men, there will be a greater proportion of older men with prostate cancer.

Studies have suggested that AAM in the USA present with higher grade and more aggressive prostate cancer. ${ }^{14}$ In Nigerian men with adenocarcinoma of the prostate, $50 \%$ had G3 tumours, which accounted for $82 \%$ of paraplegics and $52 \%$ of deaths occurring within 12 months. ${ }^{11}$

In our study, grade 1 was most common in white and coloured patients, while grades 2 and 3 were more common in black men (Fig. 2), which may indicate that black men have biologically more aggressive prostate cancer, but may also be a selection artefact as black men with poorly differentiated cancer are more likely to present with clinical symptoms, whereas those with well differentiated cancer may remain undiagnosed.

Most studies in the USA have shown that AAM present with more advanced stages of prostate cancer compared with CAM, which is attributed to racial differences in socio-economic status, education level and the availability or use of healthcare services. ${ }^{3}$ A smaller proportion of AAM than CAM take part in voluntary screening programmes and undergo recommended prostate biopsies, especially in lower-income areas. Similarly in South Africa, a lower proportion of black than white men underwent a recommended prostate biopsy. ${ }^{14}$

In African countries, PSA testing and prostate biopsy are not readily available, therefore the diagnosis is usually made clinically in patients presenting with symptoms, or histologically after surgery for suspected benign prostatic hyperplasia (BPH). ${ }^{3,9,11}$ Most black men 
in Africa present with locally advanced (41\% - 96\%) or metastatic disease $(16 \%-59 \%)$. Blacks outside Africa (e.g. in Jamaica) present with more advanced disease. ${ }^{4,11}$

In our study, locally advanced and metastatic disease was significantly more common in black and coloured than in white men (Figs 3 and 4). As in the USA, this fact may be attributed to racial differences in socio-economic status, education level and the availability or use of healthcare services. ${ }^{3}$

Studies have reported higher mean serum PSA at presentation in AAM than in CAM even after controlling for tumour stage. ${ }^{6}$ Elevated PSA at diagnosis could reflect late diagnosis owing to decreased access to healthcare or that the disease is more aggressive in AAM. Due to improved awareness and prostate cancer screening, the racial disparities in PSA appear to be decreasing in the USA. ${ }^{5}$

In our study, mean serum PSA was significantly higher in the black and coloured than in the white groups. A marked difference between the mean and median PSA values was due to a small number of very high outlier values which skewed the mean values. Therefore, median values should preferably be used to compare different groups. ${ }^{3,9}$ Mean serum PSA increased significantly according to the histological grade and clinical stage of prostate cancer. Therefore, the higher mean PSA in the black and coloured groups is explained by the higher grade and stage of prostate cancer in these groups.

AAM tend to receive potentially curative therapy less often, go untreated more often, are less likely to undergo RP, and more likely to receive RT or ADT than CAM. ${ }^{3,5,15}$ Reasons why black men are more likely to remain untreated include the desire to avoid treatment side-effects, fear, distrust of the health system, reliance on traditional healers, religious beliefs, differences in recommendations or preferences of physicians, advice from family and friends, or other information sources. ${ }^{5}$

In our study, potentially curative treatment was less often chosen by black than by coloured and white patients. Among men in whom potentially curative treatment was indicated, this was chosen by only a third of black men, compared with two-thirds of white and coloured men. Studies from the USA have suggested that there are no racial differences in mortality when there is equal treatment, suggesting that racial differences in patterns of care contribute to the racial disparity in survival. ${ }^{3}$

In our study, the mean duration of follow-up was significantly shorter in the black than in the white or coloured groups. Follow-up was also significantly shorter in high-grade than low-grade cancer, and in higher clinical stage than lower stage cancer. The most probable reason for loss to follow-up was death, indicating that the duration of follow-up can be used as a surrogate for survival. The significantly higher prostate cancer grade, stage and serum PSA in the black group can explain their shorter survival.

PSA testing to detect prostate cancer in asymptomatic men aged 50 - 70 years has led to a dramatic stage migration in developed countries. Before its introduction, approximately $80 \%$ of men presented with incurable non-localised prostate cancer. Widespread PSA testing has resulted in at least $80 \%$ of men being diagnosed with potentially curable organ-confined cancer. PSA screening has reduced prostate cancer mortality by at least $20 \%$ in Europe ${ }^{16}$ Owing to the risk of over-diagnosis and overtreatment and the costs of treatment, state-funded, population-wide PSA screening remains controversial. However, PSA testing of well-informed men at risk of prostate cancer produces a substantial stage migration towards early-stage, curable disease. Conversely, as our study demonstrates, where access to PSA screening of pre-symptomatic men is not readily available, most still present with locally advanced or metastatic, incurable prostate cancer.

\section{Conclusions}

During 1995 to 2005, black men in Western Cape Province presented with higher grade and stage prostate cancer and higher serum PSA levels than white or coloured men. Black men received potentially curative treatment less often and had a significantly shorter duration of follow-up than white or coloured men, probably owing to earlier death from prostate cancer. This demonstrates that in regions where PSA screening is not readily available and men with prostate cancer present because of cancer-related symptoms, the disease is locally advanced or metastatic (and therefore incurable) in most cases. Moreover, racial differences in stage at presentation and therapy indicate the need for greater prostate cancer awareness and education among patients and physicians. More widespread use of PSA for early detection in men at increased risk of prostate cancer and who are candidates for potentially curative therapy, is also supported.

The authors thank Mrs Mariëtte Pretorius, who maintained the prospective urological oncology database, and Mrs Santie van Deventer who typed the clinical summaries and operation reports of patients.

\section{References}

1. Parkin DM, Sitas F, Chirenje M, Stein L, Abratt R, Wabinga H. Part I: Cancer in indigenous Africans burden, distribution and trends. Lancet Oncol 2008;9(7):683-692.

Brawley OW, Jani AB, Master V. Prostate cancer and race. Curr Probl Cancer 2007;31(3):211-225.

3. Heyns CF, Lecuona AT, Trollip GS. Prostate cancer: Prevalence and treatment in African men. JMHG 2005;2(4):400-405.

4. Gueye SM, Zeigler-Johnson CM, Friebel T, et al. Clinical characteristics of prostate cancer in African Americans, American Whites and Senegalese men. Urology 2003;61(5):987-992

Powell IJ. Epidemiology and pathophysiology of prostate cancer in African-American men. J Urol Powell IJ. Epidemiolo

2007;177(2):444-449.
6. Kang JS, Maygarden SJ, Mohler JL, Pruthi RS. Comparison of clinical and pathological features in African-American and Caucasian patients with localized prostate cancer. BJU Int 2004;93(9):12071210

Delongchamps NB, Singh A, Haas GP. Epidemiology of prostate cancer in Africa: Another step in the understanding of the disease. Curr Probl Cancer 2007;31(3):226-236.

. Evans S, Metcalfe C, Ibrahim F, Persad R, Ben-Shlomo Y. Investigating Black-White differences in prostate cancer prognosis: A systematic review and meta-analysis. Int J Cancer 2008;123(2):430-435. . Heyns CF. Is prostate cancer more common and more aggressive in African men? Afr J Urol 2008;14(2): 66-74.

10. Fernandez P, De Beer PM, Van der Merwe L, Heyns CF. COX-2 promoter polymorphisms and the association with prostate cancer risk in South African men. Carcinogenesis 2008;29(12):2347-2350.

Osegbe DN. Prostate cancer in Nigerians: Facts and nonfacts. J Urol 1997;157(4):1340-1343.

12. Heyns CF, Naudé AM, Ahmed G, Stopforth HB, Stellmacher GA, Visser AJ. Serum prostate specific Heyns CF, Naudé AM, Ahmed G, Stopforth HB, Stellmacher GA, Visser AJ. Serum prostate specific
antigen as surrogate for the histological diagnosis of prostate cancer. S Afr Med J 2001;91:685-689.

Freedland SI, Sutter ME, Naitoh J, Dorey F, Csathy GS, Aronson WJ. Clinical characteristics in Black and Freedland SJ, Sutter ME, Naitoh J, Dorey F, Csathy GS, Aronson WJ. Clinical characteristics in Black
White men with prostate cancer in an equal access medical center. Urology 2000;55(3):387-390.

White men with prostate cancer in an equal access medical center. Urology 2000;55(3):387-390.
4. Heyns CF, Mathee S, Isaacs A, Kharwa A, De Beer PM, Pretorius MA. Problems with prostate specific Heyns $\mathrm{CF}$, Mathee S, Isaacs A, Kharwa A, De Beer PM, Pretorius MA. Problems with prostate specific
antigen screening for prostate cancer in the primary health care setting in South Africa. BJU Int 2003;91(9):785-788

5. Underwood W, De Monner S, Ubel P, Fagerlin A, Sanda MG, Wei JT. Racial/ethnic disparities in the treatment of localized/regional prostate cancer. J Urol 2004;171(4):1504-1507.

16. FH Schröder FH, Hugosson J, Roobool MJ, Tammela TLJ, Ciatto S, Nelen V. Screening and prostatecancer mortality in a randomized European study. N Engl J Med 2009;360:1320-1328. 